\title{
Gastro-oesophageal reflux symptoms and BMI in individuals not seeking healthcare for reflux disease
}

\author{
Katie L. Oliver ${ }^{1}$, Gloria J. Davies ${ }^{1}$ and Peter Dettmar ${ }^{2}$ \\ ${ }^{1}$ London South Bank University, London, UK and ${ }^{2}$ Technostics, Hull, East Yorkshire, UK
}

Presently, there is considerable interest about having a healthy body weight. A high body weight and BMI may influence the onset of heartburn in patients with gastro-oesophageal reflux disease ${ }^{(1)}$. This association has been demonstrated in men and women and has been reported as a determining trigger factor in the onset of symptoms and progression of heartburn to gastro-oesophageal reflux disease ${ }^{(2)}$.

The aim of the present study was to investigate the prevalence of symptoms in individuals who were not seeking healthcare for gastrooesophageal reflux disease. Ethical approval was gained from the Bedfordshire Local Research and Ethics Committee. Participants were recruited from a general practitioner surgery in Bedfordshire, UK. Subjects were screened with the use of a questionnaire and those who deemed themselves to be healthy and therefore were not seeking healthcare for gastro-oesophageal reflux disease were invited to participate. Fifty Caucasian subjects completed a lifestyle questionnaire including the GERD impact scale ${ }^{(3)}$, which is a gastro-oesophageal reflux symptoms questionnaire, and the international physical activity questionnaire ${ }^{(4)}$.

\begin{tabular}{lcccc}
\hline & \multicolumn{2}{c}{ Males $(n$ 24) } & \multicolumn{2}{c}{ Females $(n$ 26) } \\
\cline { 2 - 3 } BMI $\left(\mathrm{kg} / \mathrm{m}^{2}\right) \ldots .$. & $20-25$ & 12 & $20-25$ & 10 \\
\cline { 2 - 5 }$n \ldots .$. & 12 & & 16 & 45.0 \\
\hline Age (years) & 48.9 & 44.4 & 44.1 & 8.0 \\
Mean & 8.8 & $30-60$ & 11.1 & $34-59$ \\
SD & $34-65$ & 7 & $28-65$ & 2 \\
Range & 4 & & 8 & 26 \\
GERD impact scale reflux & & &
\end{tabular}

In males symptom onset was associated with a higher than normal BMI, although this did not reach statistical significance. Associations between BMI and symptom onset have only previously been reported in patients with gastro-oesophageal reflux disease who have a high $\mathrm{BMI}^{(1)}$, and not in those who are not seeking healthcare for the condition. Conversely, in females the highest incidence occurred in those within a normal BMI range with a history of pregnancy. This association has not previously been reported and is a new finding in females. There were moderate levels of physical activity in both males and females and each category of BMI. It would therefore seem that in these subjects the level of physical activity was of little importance in relation to the symptoms of reflux activity. In conclusion, it would seem that those not seeking healthcare for gastro-oesophageal reflux disease may benefit from education and awareness as to the aspects of diet and lifestyle, which may trigger the onset of these symptoms.

1. Nilsson M, Johnse R, Ye W, Hveem K \& Lagergren J (2004) Gut 53, 1730-1735.

2. Murray L, Johnston B, Lane A, Harvey I, Donovan J, Nair P \& Harvey R (2003) Int J Epidemiol 32, 645-650.

3. Jones R, Coyne K \& Wiklund I (2007) Aliment Pharmacol Ther 25, 1451-1459.

4. Craig CL, Marshall AL, Sjostrom M, Bauman AE, Booth ML, Ainsworth BE, Pratt M, Ekelund U, Yngve A, Sallis JF \& Oja P (2003) Med Sci Sports Exerc 35, 1381-1395. 